\title{
Labyrinthe
}

38 | 2012 (1)

Leéloquence des singes

\section{La grande scène des primates}

\section{Laurent Dubreuil}

\section{OpenEdition}

\section{Journals}

Édition électronique

URL : http://journals.openedition.org/labyrinthe/4249

DOI : $10.4000 /$ labyrinthe.4249

ISSN : 1950-6031

Éditeur

Hermann

Édition imprimée

Date de publication : 1 avril 2012

Pagination : 81-102

ISBN : 9782705682361

\section{Référence électronique}

Laurent Dubreuil, «La grande scène des primates », Labyrinthe [En ligne], 38 | 2012 (1), mis en ligne le

01 avril 2014, consulté le 01 mai 2019. URL : http://journals.openedition.org/labyrinthe/4249 ; DOI :

10.4000/labyrinthe.4249 


\title{
La grande scène des primates
}

\author{
Laurent DUBREUIL
}

Le début de cet article se situe par l'expérience d'une expérience, et mon premier séjour avec Sue Savage-Rumbaugh et ses collaborateurs. Le plus large propos, qui suit mon enthousiasme et mes doutes vis-à-vis d'une œuvre géniale et hors-normes, est bien de réfléchir sur ce théâtre physique et métaphysique, où nous demeurons, acteurs animaux dotés de la parole, accompagnés d'autre bêtes animées, plus ou moins loquaces. Travaillant depuis des années dans les replis et les failles des disciplines discursives, en particulier la philosophie et les sciences dites humaines, ou à même la littérature, je me suis engagé depuis peu sur un terrain plus scientifique. La raison avouée de cet autre voyage (le problème du langage, vu par la connaissance positive, expérimentale, et formelle) a fait de moi bizarrement le membre d'un centre d'études cognitives (à Cornell). Ici, j'essaie de jouer de ces perspectives différentes dans la poétique de mon texte, dont le récit réflexif et théorique est entrecoupé de notes, de fragments d'hypothèses formulées en vue du logos.

L. D.

Peut-être que je devrais commencer par cette scène. Je me retrouve avec des inconnus dans le grand hall intérieur d'un bâtiment aux aspects brutalistes. En face de nous se situe l'autre partie de la construction, rendue visible par de longues baies vitrées, et où réside une famille. Lorsqu'à l'automne 2010, je suis en ce lieu, à attendre pour la première fois ce qui est clairement mis en scène comme une apparition, je connais bien les différents acteurs par des livres et des films, je n'ai pas encore d'idée exacte sur le plan de ce laboratoire. Pour parvenir ce matin-là à ce qui s'appelle officiellement un trust - le terme américain, en plus de l'évidente dénotation d'un type d'entreprises, a gardé sa relation à la confiance, à la foi placée en quelques élus pour diriger un collectif - , à ce qui se nomme, avec ambiguïté, le Trust des grands singes, j'ai dû prendre un taxi depuis le centre de la petite capitale de l'état rural qu'est l'Iowa, nommée « Des Moines ». Comme cela m'arrivera par la suite, le chauffeur reste interdit quand je lui explique que je vais voir des bonobos, 
en plein cœur du Midwest, que ces singes utilisent un ordinateur pour communiquer en anglais en plus de leurs gestuelles et de leurs cris, que je suis français, que j'enseigne à Cornell University. Le Trust est un lieu sécurisé, en banlieue de la ville, sans indication d'aucune sorte, entouré de barbelés et d'un lac, avec un filtrage à l'entrée, une escorte assurée par une compagnie privée jusqu' au seuil du bâtiment lui-même, de multiples portes blindées s'ouvrant par codes et cartes magnétiques. Au-delà des premiers bureaux, les invités, accueillis à condition d'être à jour de leurs vaccinations diverses, portent un masque de chirurgien, qui protège les singes de virus humains, sans doute, et, surtout, sert de signe visuel. Je suis dans ce hall à l'occasion d'un événement mondain, pour ainsi dire. Une douzaine de personnes ont été conviées à la première présentation officielle du singe nouveau-né, baptisé Teco, le plus jeune rejeton de la famille bonobo, et qui a moins de deux mois. Cette présentation doit permettre d'autres épiphanies.

L'architecte a placé au cœur du hall une sorte de sas transparent, qui est comme un corps étranger entre la partie enclose du trust où vivent les singes - et l'espace commun aux scientifiques, aides et VISITEURS ${ }^{1}$, dont le nom vient d'être prononcé par la voix synthétique du grand ordinateur à écran tactile, où bonobos et humains appuient sur des lexigrammes en vue de composer des phrases électroniquement proférées. L'absence de perspective nette sur le dehors, l'image du sas hermétique et de l'ordinateur géant participent à une impression de science-fiction. Sciemment, ce théâtre maximise les effets d'une futurity. Ce qui s'y joue prend immédiatement valeur d'une prophétie, annonçant le temps d'un échange high-tech entre membres d'espèces différentes. Un échange en anglais, comme dans les films à grand spectacle hollywoodiens. En même temps, ce dessein SF est relativisé par la simplicité familière des rapports entretenus par les membres de la petite tribu. Ce qui est science dans le discours de Sue Savage-Rumbaugh, le savant responsable de l'expérience, n'est pas plus fiction que chez d'autres psychologues expérimentaux, les spécialistes du cerveau humain par exemple; et pas davantage dans l'onirique, dans l'improbable demain. Puis Kanzi l'aîné, Panbanisha sa sœur, et l'adolescent Nyota, lorsqu'ils passent le sas, ne s'apprêtent pas à décrire la vie du vingt et unième siècle. Ils essaient plutôt de se faire

1. Conformément à l'usage, je mets en capitales certains mots correspondant aux lexigrammes. 


\section{La grande scène des primates}

entendre, ils en profitent pour une doléance, une demande d'amélioration, un commentaire, ou une plaisanterie.

Après l'appel, Panbanisha et son fils Nyota arrivent au sas; Sue avec eux, qui porte contre elle Teco, le jeune rejeton de Kanzi, délaissé par sa mère (sauvage et que l'on dirait biologique). Les gens autour de moi sont tous des habitués du trust, d'une manière ou d'une autre: des voisins, des donneurs potentiels, la photographe attitrée, une femme de lettres auteur de romans à succès et qui vient de s'inspirer du laboratoire pour son prochain produit. Tous savent qu'une façon de bien commencer une telle session est de jouer un peu avec les singes, à CHASE \& TICKLE essentiellement, quelque part entre la course, le cache-cache et les chatouilles (en l'occurrence virtuelles, par paroi de verre interposée). Ce qui veut dire au passage que le précieux sas doit être rouvert, afin de permettre à Nyota, l'expert actuel du tickling, d'arpenter son enclos avec, de l'autre côté, ses compagnons humains du jour. L'encadrement architectural et la mission du trust semblent en porte-à-faux avec les génies du lieu. La conversation qui suit, ponctuée de cris, de gestes déictiques, des méta-commentaires de Sue, est assez brouillonne. Nyota et Panbanisha demandent avec entêtement à aller DEHORS, dans cette portion du terrain extérieur qui est alors condamnée par suite d'une inondation (EAU GROSSE GROSSE, dit Sue). Nyota réclame son JUS ; Panbanisha souhaite boire le CAFÉ du BUREAU DU STAFF. Personne ne tient vraiment en place, sauf Teco, très pacifique. Une fois les boissons consommées, et les SURPRISES déballées (offrir des cadeaux est le plus sûr moyen de se faire accepter par la famille simiesque), Nyota et Panbanisha ont suffisamment passé de temps avec des personnes qu'elles aiment ou connaissent plus ou moins, avec qui aucun contact physique n'est possible, et qui sont vouées à s'éclipser pour des mois, des années. Cette partie de la célébration est terminée. Sue quitte ensuite le sas et l'enclos, pour parler dans le hall de Teco, à qui elle prodigue des soins maternels exceptionnels, tout en délivrant une explication biologique et comportementale des phénomènes. La baby shower s'achève bientôt.

- Le langage est fuyant. - Nous disons moins et plus que nous ne pensons, c'est-à-dire autant. - Les mots sont des organes-obstacles. - Nos discours sont ouverts aux quatre vents. - Le langage organise le réel, à quelques défauts près. - Ce que j'écris est rempli de contradictions. 
Des singes se trouvent sur terre, en plusieurs points de cette planète, ou y résidèrent, qui expriment quelque chose par le biais d'un système de langage humain. Non seulement ces animaux sont capables d'identifier des mots, de les rapporter à un référent, d'en déduire pragmatiquement un signifié - ce qu'accomplissent aussi, en marge des humains, bon nombre d'individus issus d'autres espèces que primates ${ }^{2}-$, ils perçoivent parfois un ordre dans l'énoncé, et ils entrent dans des conversations, souvent à leur tour, maniant des paroles, qui informent, décrivent, répondent, qui requièrent, qui ont une action. Or ces singes ne sont pas humains: ils sont gorille, orang-outang, chimpanzé, bonobo. Très peu vocalisent; beaucoup utilisent des variantes de la langue des signes américaine; quand les autres ont recours à des photographies représentant des objets, à des signes arbitraires en plastique, à des touches d'ordinateurs. Ils ont presque tous reçu cette instruction au contact de scientifiques. Ils vivent, vécurent ou vivront dans des zoos, des sanctuaires, des laboratoires, des maisons. Depuis les années dix-neuf cent quarante en particulier, ils ont fait l'objet d'articles, de livres, de films ${ }^{3}$, de forums, de colloques - de recherche, de vulgarisation, de publicité pour la bonne cause, de dérision. Nous venons aujourd'hui après plusieurs décennies d'instruction, d'insuccès, de réussites. Le débat n'est pas tranché, quoique soient désormais de rigueur un scepticisme, volontiers sardonique, parmi les savants ${ }^{4}$, et une surprise enthousiaste et désespérément répétitive, dans les médias. Pour moi, la question de savoir si certains singes comprennent et utilisent à leurs manières une ou des langues humaines ${ }^{5}$, si, donc, ces animaux ont une relation interprétative et productrice avec «le »

2. Le cas du chien Rico a récemment suscité quelque émotion en ce sens. Cf. Juliane Kaminski et al., «Word Learning in a domestic dog », Science, 11 juin 2004.

3. Oui, de films aussi, qui, très tôt, dépassèrent le stade du simple « documentaire scientifique » restreint à la communauté des savants (cette dernière tradition étant inaugurée par les époux Kellog, qui conservèrent des images de la petite Gua, chimpanzée enfant élevée chez eux). Cela va de Barbet Schroeder (Koko, le gorille qui parle, 1978) au récent Project Nim de Jim Marsh (2011).

4. Cf., pour une version typique, l'article de Jon Cohen, paru dans la partie « magazine » de la revue Science: «Boxed about the ears, apes language research is still standing », 2 avril 2010.

5. Les deux « langues » dominantes sont l'anglais et le langage des signes américains (ASL), dans une version généralement simplifiée, et adaptée à la physiologie des primates. À l’Université de Kyoto, Ai, une femelle chimpanzé, et d'autres membres de la même espèce, ont, en plus des mêmes lexigrammes utilisés au Trust, recours à des caractères kanji pour désigner des objets ou des qualités (comme des couleurs). Pour une vue synthétique sur cette tentative, lire Tetsuro Matsuzawa, « Chimpanzee intelligence in nature and in captivity », ch. 15 de Linda Frances Marchant \& Toshisada Nishida (dir.), Great Ape Societies, Cambridge (G.B.), Cambridge University Press, 1996. 


\section{La grande scène des primates}

langage, cette question ne se pose plus guère. Je songe en particulier aux bonobos du trust. D’un point de vue métrique, même revus à la baisse (ainsi que le souhaiteraient certains), les résultats de l'étude menée en 1993 par Savage-Rumbaugh sur la compréhension comparée de Kanzi et d'un enfant humain ${ }^{6}$ demeurent: bien au-delà du hasard, un bonobo est capable d'analyser des énoncés sur la foi d'une décomposition syntaxique et sémantique, puis de réaliser les actes requis, avec une marge d'erreur approchant le niveau d'un petit d'homme. D'un point de vue subjectif, de la même manière que nous pensons entre humains conversant au café que nous sommes bien en train d'échanger sans avoir besoin de vérifier nos facultés au préalable, ni d'obtenir les preuves vacillantes que nous nous comprenons vraiment, il suffit d'être dans un rapport un peu long avec Kanzi et sa famille - au-delà de la mise en spectacle du sas d'observation - pour perdre tout doute sur le fait que nous sommes parfois, et avec eux, immergés ensemble dans des discussions. De drôles de discussions, d'accord. Mais je ne retire aucun des mots que j'ai employés; car j'entends que vous reveniez sur eux, et qu'ils signifient ce qu'ils peuvent pour vous.

Le sémantisme: produit par les mots dans leurs agencements, et produisant les mots en s'y arrêtant; streams of consciousness. Les mots sont repères, aide-mémoires (il en est d'autres), qui servent à « fixer les idées » ainsi acquises et à venir. Fixant, ils altèrent. Le principe structurant des mots permet, par cet effet, l'émergence d'autres tâches cognitives, même non verbales, en ce qu'il organise le pensé. Les mots sont des attracteurs et des émetteurs de sens. Mais le sémantisme n'est pas le sens.

Décidément, les questions suscitées par l'expérience des singes langagiers et langagés ${ }^{7}$ valent davantage dès qu'est dépassée la stérile controverse sur une définition catégorielle excluant d'avance tels individus du domaine discursif ou les y intégrant de force. Qu'est-ce qu'une relation au langage humain permet et empêche, dans la cognition et l'action? Quelles analogies se déduisent de l'accession au logos chez

6. Sue Savage-Rumbaugh et al., Language Comprehension in Ape and Child, in Monographs of the Society for Research and Child Development, LVIII. 3-4, 1993.

7. Je reprends l'expression de Sue Savage-Rumbaugh (languaged apes). Michael Tomasello parle, lui, de « singes "linguistiques" ». 
l'humain préhistorique? Quels liens établir entre les divers systèmes de communication animale? Quel « propre » de l'humain pourrait-il rester en notre propriété, à continuer sur cette voie ? Voilà de plus intéressantes perspectives. S'y associent les réflexions éthiques, inévitables, sur le modèle si... alors... : si des singes peuvent dire leurs sentiments, alors qu'en déduire sur notre manière de les traiter? Toutes ces interrogations vont rapidement de la querelle sur la personnalité juridique des primates (garantie jusqu'ici par les seules îles Baléares) au végétalisme, se voulant préserver de blesser tout «être sentient ${ }^{8}$ », en vertu d'un passage qui établit une continuité animale dont l'homme, soudain, deviendrait le protecteur particulier, et que ces bonobos de laboratoire illustreraient à regret. De tout cela, j'aimerais à mon tour parler, en détail, sachant que me manqueraient la plupart des réponses. Ce serait pour plus d'un livre; l'un d'eux s'intitulerait La Métaphysique à Kanzi.

Pour l'heure, j'en veux rester aux réflexions préparatoires. À la fois, que des singes usent de l'anglais, de leurs mains, de leurs voix, pour dire et vouloir dire constitue une forme d'événement total, dont les répercussions conceptuelles, pratiques et politiques sont immenses. À la fois, cet événement n'apparaît presque pas comme tel: pour les uns, il fut tellement préparé par toutes les fictions animalières (où entrent les sciences avec les «mythes ») que le choc s'aplatit en un bien sûr, je m'en doutais, nous l'avons toujours su, d'ailleurs mon chien etc.; pour les autres, il est savamment nié d'avance et recouvert ensuite comme supercherie, boniment, racontar, allusion rituelle. [Sur ce dernier point, il suffit de voir, parmi les « Paroles de singes » de ce dossier, que Daniel Dennett, qui est athée, en comparant une conversation avec Dieu et un échange avec Kanzi, place celui-ci au niveau d'une inexistante déité ${ }^{9}$; le bonobo vit pourtant bel et bien, quelles que soient ses capacités verbales. Par ironie, Chomsky imagine aussi la prière du chimpanzé $\mathrm{Nim}^{10}$, à qui il dénie toute compétence langagière, à un Dieu absent. Comme il est étrange que ces bons mots, aux alentours du religieux - la bête et le souverain, décidément ${ }^{11}$ - occasionnent plus d'une approximation. La plupart des opposants à la possibilité de singes éloquents, et qui décèlent

8. Je fais ici allusion aux mouvements anti-spécistes, pour qui Peter Singer reste une référence; cf. son Animal Liberation, New York, Avon Books, 1975.

9. Texte [11] de cette anthologie.

10. Texte [9].

11. Jacques Derrida, Séminaire. La Bête et le souverain, Paris, Galilée, 2 vol., 2008-2009. 


\section{La grande scène des primates}

apparemment un rapport occulte entre ces recherches, les récits sur les ovnis, les religions, j'en passe ${ }^{12}$, ne font plus que citer la citation de la citation; avec le psittacisme associé. Bien sûr, dès lors, la chaîne de ces on-dit lie surtout les anneaux d'une phraséologie dont le but social est clair (comme: ne donnez plus d'argent à ces biologistes insensés, versez plutôt les fonds aux adeptes de la linguistique générative). Le problème, lui, est esquivé.]

Pour moi du moins, ces singes qui parlent et dont on parle font événement. Ils ne transforment pas mon existence du tout au tout, ni ne remettent en cause « ce que j' ai toujours cru » ou ce que je pourrais croire que « nous » croyons. Non, il ne s'agit pas du début d'une ère nouvelle, ni de l'évangile selon Teco. Mais un événement, pour ce qu'il nous donne, et par ce que nous en construisons, confirme, modifie, ouvre. Le présent texte, de la sorte, répond à l'émergence subjective d'une événementialité dans la distribution et l'édiction de la parole; il voudrait en faire ressentir l'acuité, avant - qui sait? - d'en redire plus tard la destination, la portée.

Dans le cas du langage, les neurosciences nous mettent face à des faits statistiques, pas face à la signification: on s'intéresse à la plus grande fréquence dans un certain contexte. Les dictionnaires de corpus raisonnent eux sur l'advenu (sous une forme très limitée).

Une relative transparence de l'énoncé est produite par le contrôle qu'exerce chaque sujet sur soi-même et par les limites conventionnelles, qui vont des plus générales aux plus particulières (c'est-à-dire: de l'ensemble morphologiesyntaxe-phonologie-prosodie... aux régimes discursifs, conventions sociales, etc.). Mais le processus de verbalisation et de compréhension est source d'opacité. II y a donc bien du partageable entre les mots, sauf qu'on en surestime l'ampleur trop souvent, ou que - par un fatalisme - on en vient à l'effacer d'un coup au nom de la discordance.

Je vais trop vite. Sans quoi je serais trop ralenti, pensé-je. Mais quand même, ne devrais-je pas revenir sur cette extraordinaire affirmation, sur la justesse de cet «événement »? Puis-je à ce point passer sous silence la myriade d'arguments contraires, visant à annuler le bien-fondé de ces

12. Je pense ici à l'article commandé à Clive Wyne («Aping language ») par la revue Skeptic (31 octobre 2007), qui est spécialisée dans la dénonciations des miracles, des soucoupes volantes, etc. 


\section{Labyrinthe, $n^{\circ} 38$}

nombreuses expériences? L'envie ne manque pas. Je vais être schématique, ce qui vaut peut-être mieux, ce qui tendrait à éviter l'embourbement.

Une première série de questions méthodiques est identifiable, qui appartiennent aux procédures de vérification propres à toute expérience scientifique. Ces interrogations sont: (1) mesure-t-on vraiment une performance linguistique en demandant à un singe d'accomplir un ordre proféré, ou de faire le lien entre un objet et son nom? (2) ne risque-ton pas de surinterpréter? (3) est-ce que tout cela est reproductible? Le doute sur la mesure est $a$ priori le plus radical, en particulier parce qu'il se réfère à une scène constitutive de la psychologie moderne - lorsqu'en 1907, Oskar Pfungst montra que Hans, « cheval savant », arrivait à compter juste en analysant l'attitude physique de son entraîneur, et non par suite de sa propre maîtrise de l'arithmétique. En somme, les tests sur les singes éloquents pourraient se limiter à la mesure de leur capacité à « lire » les visages des expérimentateurs et/ou reproduire les sons ou les gestes. Or cet effet, habituellement nommé «Clever Hans », est si notoire que, depuis les années 1960 et les travaux des époux Gardner au moins, chacun s'emploie à l'éviter. Le chimpanzé Washoe [45] désignait avec exactitude à un observateur, qui, lui, ne connaissait pas directement la réponse exacte, des objets qui lui étaient montrés par un tiers. Kanzi, en réalisant physiquement les commandes orales de Savage-Rumbaugh, qui étaient souvent absurdes (comme « Mets les aiguilles de pins dans le réfrigérateur » ou « fais une piqûre au chien en peluche »), ne voyait pas le visage de la scientifique, caché par un masque de soudeur ${ }^{13}$. Tous ces singes sont à même, à des degrés divers, d'interagir verbalement avec des gens qu'ils connaissent peu, et en l'absence de leurs « entraîneurs ».

Le problème de la surinterprétation est notable. La plupart des primates n'ayant pas la possibilité anatomique de bouger leurs poignets comme le font les humains, le recours au langage des signes nécessite une forme d'adaptation - et un décodage. L'idée de Duane Rumbaugh, avec les lexigrammes et ensuite l'ordinateur parlant, consistait à surmonter l'accusation. Il demeure envisageable que certains énoncés soient compris très "généreusement » (et l'anthologie en délivre des exemples). Il est toutefois frappant de voir que les psychologues les plus exigeants à l'égard des séquences verbales utilisées par les singes sont soudain d'un merveilleux laxisme avec les humains. Ainsi, Michael Tomasello, fort

13. Protocole utilisé pour la préparation de Language Comprehension in Ape and Child. 


\section{La grande scène des primates}

réservé sur le cas des primates loquaces, n'hésite pourtant pas à gloser ses conspécifiques avec une grande libéralité: un bébé de treize mois qui babille et pointe du doigt un arbre de Noël veut dire « Regarde le sapin de Noël ! C'est super, hein ? ${ }^{14} »$; un adulte qui désigne de l'index le stylo dépassant d'un livre signifie "Regarde, le stylo est dans une situation précaire; fais attention, s'il te plait, et ne le laisse pas tomber ${ }^{15} »$. Dans ces exemples, et tant d'autres, le double standard est patent. Si un chimpanzé signe MANGER POMME, cela ne prouve pas grand-chose; si un humain bouge le petit doigt, une paraphrase déplie immédiatement les complexités sémantiques. C'est qu'en fait, la présupposition d'un univers linguistique partageable est contenue dans l'interprétation. À ce titre, les énoncés des singes langagés sont en effet susceptibles de gloses excessives; comme ceux des humains. Malgré les proclamations d'objectivité scientifique, la lecture contient en préalable une donation subreptice de possibilité. L'éventuelle déformation sémantique, ou l'austérité de l'interprétation, dépendent de l'admission sous-jacente d'une (in)capacité potentielle. Retour à l'envoyeur.

La reproductibilité est autre chose. Tandis que chaque test a été répété avec chaque individu, et qu'en tout plus d'une douzaine de singes ont utilisé des formes de langage humain, les nombres restent faibles. Mais qui veut vraiment se lancer à la suite de ces tentatives? Puis, là encore, il serait intéressant de se demander pourquoi la plupart des expériences en neuro-imagerie (qui concernent souvent une quinzaine d'individus, en général des étudiants de premier cycle d'université américaine) sont, elles, jugées immédiatement si crédibles et profondes.

Se pourrait-il que ces singes éloquents qui ne sont humains aient un recours parcimonieux au langage? Plus ils fréquentent quelqu'un, plus ils lui parlent (Nyota, adolescent d'action et peu disert, au bout de quelques jours, lorsque nous jouons à nous cacher le visage, qui commente spontanément et pointe vers MASQUE sur le clavier). Je ne les ai pas vus parler pour « briser la glace ». Comme si leur rapport, assez intense, à l'anglais sous ses formes de sons, de lexigrammes, de lettres, restait plus adventice. II leur faut plein d'activités différentes pour avoir envie d'en dire quelque chose; sinon, ils se renferment. Se parlent-ils en leur for intérieur?

14. Michael Tomasello, Origins of Human Communication, Cambridge (É.-U.), MIT Press, 2008, p. 114. 15. Id., 64 . 
Les objections les plus valables tiennent aux limitations cognitives et à la définition du langage. Pour fameuse qu'elle soit, l'idée que les singes en restent à l'imitation, à la simple reproduction des signes et des mots ne tient pas. Herbert Terrace popularisa ce motif, après avoir échoué à enseigner le langage des signes au singe Nim Chimpsky. L'erreur était surtout la sienne (empêcher un attachement affectif entre les expérimentateurs et $\mathrm{Nim}$, confondre enseignement et répétition de gestes conditionnés, prévenir de facto Nim de s'exprimer), le blâme en revint aux chimpanzés. On peut lire, dans un exemple des « Paroles de singe », les conversations tragi-comiques entre Nim et ses « enseignantes » [8]. Il y apparaît que Nim n'imite pas forcément plus les humains que l'inverse, car cette éducation par réitération gestuelle fait circuler encore et encore les mêmes mots, selon un ordre au moins aussi aléatoire chez les locuteurs humains qu'avec le chimpanzé. Enfin, s'il est vrai que beaucoup de discussions avec des grands singes sont « aidées » par les propos précédemment proférés, des occurrences spontanées sont aussi repérables dans les expériences les plus réussies.

On atteint en revanche de meilleurs problèmes sur le plan de la syntaxe et de la récursivité (ce dernier trait étant devenu, à la suite d'un article célèbre paru en 2002 dans Science, le camp retranché du privilège humain sur le langage chez Chomsky et al.). Les singes éloquents ont-ils démontré une maîtrise syntaxique comparable à celle d'un universitaire? non. Sont-ils capables de percevoir avec exactitude des phrases comme «Emmène dans la chambre la balle qui est sur le pot ${ }^{16}$ »? peut-être pas. Mais où commencent la faculté du langage et sa maîtrise? Puis, comme le soulignent certains auteurs, quels humains se montrent tout à fait à l'aise avec une récursivité dès qu'elle passe un certain degré ${ }^{17}$ ? $\mathrm{N}$ 'aurions-nous donc pas affaire à des niveaux d'intensité pour la faculté langagière? Les singes qui signent, utilisent les lexigrammes ou vocalisent, démontrent une compréhension supérieure à leur production. Il

16. Je reprends un exemple donné par Michael Corballis, qui suppose qu'une telle phrase « s'avérerait impossible » à comprendre pour Kanzi (« Recursion, language, and starlings », Cognitive Science XXXI-4, 2007, § 5). Je note que le simple fait de traduire la phrase en français nécessite un réagencement syntaxique: «dans la chambre » est dans l'orignal to the bedroom, la préposition to exprimant un directif, que seule la place du syntagme rend sensible en français.

17. Corballis souligne à juste titre qu'une récursivité centrale devient difficile à manier pour les humains dès le quatrième degré, cf. id., § 4. La chose est sensible pour les spectateurs d'Inception, le film de Christopher Nolan (2010). 


\section{La grande scène des primates}

est rare que chimpanzés ou les bonobos combinent plus de trois mots, et beaucoup de leurs énoncés se limitent à un ou deux termes. Une capacité grammaticale est mobilisée, même si elle paraît différente de la syntaxe qu'ils reconnaissent par ailleurs ${ }^{18}$. Mais est-il si surprenant de constater des limitations dans l'accès au langage chez des individus d'espèces n'ayant manifestement pas développé de système de communication comparable en sophistication à celui des humains ${ }^{19}$ ? Des analogies viennent encore à l'esprit. Dans les autres langues dont nous, humains, pouvons disposer au-delà de l'idiome maternel, nous comprenons en général mieux que nous ne parlons ou écrivons. Quant à la manipulation correcte des mots, elle est graduelle, hétérogène selon les âges, les locuteurs, les situations, l'éducation, les capacités cognitives. Les enfants autistes, les sujets mentalement déficients, voire les illettrés ont des points d'arrêt dans leur expression que d'autres ignorent. Bref, il est plus que probable que les singes dont je parle me soient très inférieurs dans leur compréhension, leur manipulation de l'anglais (alors même qu'en aucun cas je ne dispose dans cette langue de la facilité que me permet le français), mais plus d'un homo loquax, malgré tout, ne s'exprime pas comme je fais. Il y aurait encore à dire, potentiellement, sur les particularités de chaque espèce, de chaque individu - ainsi que sur les types d'enseignement. Ainsi, les gorilles Michael et Koko, élevés par Penny Patterson, semblent disposer d'une expressivité très grande, à proportion d'une grammaire fort difficile d'accès. Sue Savage-Rumbaugh estime aujourd'hui qu'une part notable des limitations linguistiques chez Kanzi, Panbanisha et Nyota dépendent des conditions d'acquisition, de leur environnement « culturel » mixte; les progrès de Teco pourraient aider à valider ou infirmer cette intuition.

En plus de ces blocages intensifs, le recours au langage humain par les singes est lui aussi particulier. Usage parcimonieux, surtout jussif ou « orienté » par une demande d'objet, quoiqu'à mon avis, les énoncés descriptifs voire narratifs soient plus présents qu'on ne le rapporte, en tout cas pour la famille $\mathrm{Wamba}^{20}$. Dans ces bornes pourtant, ces animaux non humains se réfèrent à des événements passés, indiquent leur état d'esprit,

18. Sur ce point, Tomasello, Origins of Human Communication, ch. 6.

19. Pour les systèmes de communication chez des primates « inférieurs », voir les travaux de Cheney et Seyfarth, ainsi que ceux de Zuberbühler; cf. [6] et [49].

20. Pour un début de discussion à ce propos, cf. Heidi Lyn, « Nonhuman primates do declare! », Language \& Communication, XXXI-1, 2011. NB: Wamba est le «nom de famille» des bonobos élevés par Sue Savage-Rumbaugh, rendu célèbre depuis l'article qu'elle a cosigné avec eux, à la grande 
évoquent une situation, inventent une histoire, expriment une inquiétude, communiquent un désir, perçoivent le juste et l'injuste, renvoient à des idées abstraites par des vocalisations, des gestes, le truchement d'une voix synthétique, l'écriture de signes à la craie ${ }^{21}$. Et comprennent bien davantage. Peut-être que cela ne prouve nulle maitrise absolue du langage, et que, comme l'écrivait quelqu'un, Kanzi serait incapable de participer à un forum sur internet sur le rapport entre le langage et la conscience ${ }^{22}$ (j'attends que la métaréflexion conceptuelle soit désormais édictée comme élément clé de la parole).

J'ai néanmoins du mal, ici, à oublier qu'existe une tradition politique et théorique d'exclusion se réclamant d'arguments identiques, afin de maintenir en minorité une part notable de l'humanité. Pour justifier la servitude, et tandis qu'il associe la tenue de la rationalité discursive à la seule animalité humaine ${ }^{23}$, Aristote pose le cas mixte de «l'esclave par nature » qui « est dans la communauté du logos, dans la mesure où il peut le percevoir mais sans l'avoir ${ }^{24} »$. Et quand des soi-disant sceptiques assurent que proférer des mots, former des signes ne prouve rien de l'idéation sous-jacente et n'indique aucune conceptualité, je ne parviens pas à oublier la psychologie coloniale, de David Hume à Gustave Le Bon, qui avait d'identiques scrupules à l'égard des Nègres. Hume: «En Jamaïque, pour tout dire, on parle d'un Nègre qui serait un homme de talent et d'études; mais il est probable qu'on l'admire pour de bien minces exploits, comme un perroquet prononçant quelques mots ${ }^{25} »$. Le Bon: «On fait aisément un bachelier ou un avocat d'un nègre ou d'un Japonais ; mais on ne lui donne qu'un simple vernis tout à fait superficiel, sans action sur sa constitution mentale et dont il ne saurait tirer aucun parti. [...] Lorsque des peuples sont différents, les mots considérés chez

consternation de la profession (« Welfare of Apes in Captive Environments », Journal of Applied Animal Welfare Science, X-1, 2007).

21. Sur le juste et l'injuste, lire Heidi Lyn et al., « Precursors of morality », Language \& Communication, 28 (2008). Dans Kanzi II (second film produit par NHK, 2000), on peut voir Panbanisha écrire à la craie des lexigrammes dans un but perlocutoire.

22. Réflexion de Stevan Harnad, dans sa participation au forum à l'URL http://www.onthehuman. org/2011/01/human-language-human-consciousness/.

23. Aristote, Politique I, 2, 1253a.

24. Id. I, 5, 1254b.

25. David Hume, « On National Characters » I, 21, n. 1, in Essays. Moral, Political and Literary, Oxford, Oxford University Press, 1963,p. 213. Ajoutons que Irene Pepperberg, avec Alex le perroquet, a démontré chez cet oiseau des capacités de compréhension et d'utilisation signifiante des mots humains proférés. Cf. Pepperberg, The Alex Studies, Cambridge (É.-U.), Harvard University Press, 1999. 


\section{La grande scène des primates}

eux comme correspondants représentent des modes de penser et de sentir tellement éloignés qu'en réalité leurs langues n'ont pas de synonymes et que la traduction de l'une à l'autre est impossible ${ }^{26} »$. - Rien ici ne démontre qu'il en va de même pour les primates éloquents que pour les barbares asservis, ou les Noirs déportés. La conformité des arguments rappelle cependant, et à toutes fins utiles, l'extrême condensation politique des lignes de partage dans et autour de l'humanité; quelles que soient les intentions ou les méthodes.

Réflexion dans un article stupide du New York Times à propos d'une rencontre entre Panbanisha et un journaliste: on dirait une scène tirée de Beckett. Ça se veut dévastateur (l'auteur corrige d'ailleurs en " ou des Marx brothers"). Or non. Quand Vladimir et Estragon échangent des mots par exemple [E. Je savais que c'était lui. V. Qui? E. Godot. V. Mais ce n'est pas Godot. E. Ce n'est pas Godot!V. Ce n'est pas Godot. E. Qui c'est alors? V. C'est Pozzo.], ne parlent-ils pas? La négativité du langage est exhibée par Beckett. En même temps, cette vacuité, ce minimum sont le maximum de vie pour ces personnages qui, tant qu'ils continuent, existent. Leçon parfois identique chez les singes à paroles: ce rien, ce pas grand-chose, ce dérisoire, c'est ce que nous sommes tous, et c'est de là que nous partons.

Quand j'ai commencé la recherche, je ne voulais guère travailler sur le langage. Je m'intéressais surtout à ce qu'il permettait, formait, transcrivait : la littérature, la description de la possession, l'amitié, la réflexion, les sentiments ; j'avais, sinon, désir de ne pas trop m'y appesantir. Comme beaucoup de gens dits « de ma génération », j'avais envie de ne pas rajouter au déluge de volumes sur le «tournant linguistique ». Cependant, je ne croyais pas à l'activisme du fait pur, au cognitif immuable sous sa qualification discursive, à la réalité absolue de l'équation. Si, peu à peu, le langage est devenu l'un de mes « objets », il ne fut jamais mon enjeu. J'ai œuvré en ce sens afin d'éviter de rester captif du cercle du dire. Je pense maintenant que le cercle est ouvert de toute façon; et que je tentais de localiser toutes ces ruptures, en commençant par elles, et devant revenir au logos. Oui, je travaille sur le langage, un peu forcé, opiniâtre. Ayant envisagé comment l'on pense ce qu'on dit et vice versa dans les disciplines discursives du savoir (une grande part du travail mené au sein

26. Gustave Le Bon, Les Lois psychologiques de l'évolution des peuples, Paris, Alcan, 1894, p. 35 et 93. 
de Labyrinthe), certains usages du pouvoir dans la constitution de nos mots, quelques ressources intellectives, épistémiques de la mobilisation langagière littéraire, j' ai entrepris de m'engager dans un nouveau rapport avec les sciences. De voir singulièrement si des propriétés induites par le fait du langage humain (telle la défectivité) lui étaient réservées, ou découlaient d'autres dispositions, physiques ou métaphysiques. Étant concédé - cela se peut refuser - qu'existe un langage humain verbal, quelles aventures lui arrive-t-il lorsqu'il est déplacé ? Lorsqu'il est observé depuis l'intérieur de la boîte crânienne, la surface du néocortex et l'épaisseur du cerveau, tous lieux où il ne séjourne qu'à demi. Lorsqu'il s'effectue, partiellement réinventé par contraintes, entre des robots qui apprennent à faire du sens, à transmettre des rudiments d'information ${ }^{27}$. Lorsqu'il se compare à la propagation d'un signal, à la diffusion visuelle de renseignements sur un état biologique, aux avertissements proférés par des animaux à l'égard de leurs compagnons. Ou lorsqu'il est manipulé par quelques singes.

Comprendre le langage pour «l'Humaniste » que, par le biais des traductions, je suis devenu professionnellement aux États-Unis ${ }^{28}$, menait à l'examen de la parole une fois déplacée. Et pour l'anti-humaniste que je suis depuis l'enfance (sous ses versions primitive, sardonique, bavarde, pédophobe et post-postmoderne), comment ne pas avoir envie de rencontrer des locuteurs inhumains? Sur ce, accordons-nous un peu. Par les motifs du pouvoir, de l'indiscipline, du littéraire, du langagier, le souci demeure bien de comment penser et vivre, leur quid et leur quod, vivre sa pensée, penser sa vie. Tout le reste de la recherche intervient en fonction de cela, dirais-je; perdre de vue ces vastes questions est la tentation permanente, consolatrice; tant pis pour ceux qui en restent là. Tant mieux si des bonobos m'aident sur ce chemin. Je sais qu'eux aussi commencent à s'inquiéter de tout cela, et sûrement plus que la plupart des humains.

27. Je fais référence aux expériences conduites par Luc Steels et ses collaborateurs au Sony Lab, à Paris. Pour une présentation générale, voir http://www.talking-heads.csl.sony.fr.

28. Je préfère la dénomination de littéraire, et de disciplines littéraires, à humanist et Humanities; mais je dois aussi vivre avec ces labels. 


\section{La grande scène des primates}

Le langage n'est pas un cercle vicieux, il est déjà ouvert.

Monte une angoisse. Ce « déplacement » du langage, sous ses dehors perturbateurs, n'est-il pas en fait le comble de l'anthropocentrisme? Regardez. Vous choisissez des singes pour votre investigation. Des primates, comme les humains, heureux hasard! Sans compter que les « meilleurs candidats » sont les espèces les plus proches génétiquement de la lignée Homo - les grands singes, et, au sein de leur groupe, de préférence les deux branches de Pan, les chimpanzés et les bonobos. D'une part, vous privilégiez les espèces les moins in-humaines. D'autre part, vous projetez déjà des caractéristiques étrangères sur d'autres êtres vivants. Un peu plus loin, ignorez-vous que plus d'un grand primatologue agite, afin de promouvoir ses pratiques, toute une rhétorique de l'utilité pour les hommes de mieux se comprendre eux-mêmes via les singes? N'est-ce point préjuger à l'excès? Vouloir transférer enfin le langage humain à des animaux, si sophistiqués soient-ils intellectuellement, équivaut à délaisser d'entrée de jeu, ou à minimiser, des modes cognitifs et affectifs nonpareils, à les faire disparaître sous un étalonnage, un devenir-humain, qui, en général, est un devenir-américain dans un laboratoire prison.

Je saisis tout à fait. Ces arguments me paraissent pourtant demi-habiles dans l'ensemble. Car les solutions alternatives sont également anthropocentriques. Qu'il y ait absolument un domaine réservé des humains? C'est ce que cherchait à combattre Darwin, lorsqu'il identifiait dans son entreprise la voie vers une « métaphysique » pensant les « babouins $»^{29} »$. Dommage que tant de ses héritiers proclamés, au nom de l'évolution, reprennent la litanie des propriétés humaines. Refuser toute compétence au regard du langage humain chez d'autres espèces n'est pas moins anthropocentrique que la reconnaissance d'une potentialité, peut-être favorisée par un système de communication d'ores et déjà syntaxique, sémantique et morphologique. Qu'un biais se fasse ressentir en faveur des autres primates parmi les chercheurs en cognition animale, comment le nier? Je rêve de plus d'efforts conjoints avec des perroquets, des corbeaux, des éléphants, des dauphins, des cachalots, et, près de nous, des chiens, des ânes. Tous pensent beaucoup; me touchent, et me parlent. Mais je m'étonne de la défiance spéciale qui atteint les primates en attendant. Derechef, exclure notre lignée correspondrait à un désir d'exotisme

29. Charles Darwin, Notebook M, p. 84e, reproduit à l'adresse http://darwin-online.uk.org. 
épistémique qui nous assimile à nos autres. Alors je peux déplorer le systématique retour à l'humain des primatologues; et je «ferai avec".

Le reproche au sujet du transfert du langage est le plus difficile, $a$ fortiori lorsqu'est postulé que, par les mots d'un individu, nous disposons d'une fenêtre ouvrant immédiatement sur l'esprit d'une espèce ${ }^{30}$. Mais les autres solutions me gênent à leur tour. Ne plus se poser de question, construire des problèmes de type mathématico-logique pour mieux appréhender cette cognition animale, cantonner d'avance les autres primates dans l'affect ou l'instinct et suspendre une communication différente - rien ne me va, j'avoue. Puis, quelles que soient les bases cérébrales dont les humains disposent, aucun d'entre nous ne parlait non plus à la naissance; il a fallu du transfert. C'est juste que je crois délicat, et jusqu'ici inédit, que des humains forment des discours et fondent des actions échappant entièrement à l' anthropocentrisme. Choisir son genre de perversion, en somme...

La représentation d'un langage parfait, bien plus qu'il n'est. Toutes nos hésitations à l'oral, nos fautes, nos repentirs, nos ellipses, nos aposiopèses. Tout ce que l'écrit à son tour réalise sans le communiquer, et vice versa. Tout ce que l'expression littéraire montre de signification dans la déchéance et l'excès de sens. Continuez après cela de renvoyer à ce calque des idées, à cette forêt d'arbres syntaxiques, à ces catégories définitoires et formelles. Mais de quoi parlez-vous?

Il est vrai qu'enseigner à des générations de bonobos à manier des lexigrammes, à comprendre l'anglais et à s'exprimer avec des étrangers dans un laboratoire est une folie; et l'on sort du rang des humains à procéder ainsi. Il n'est pas du tout certain à mes yeux que cette hubris soit recommandable. Seulement voilà, comme dans une tragédie, la démesure est là, les destins se sont enclenchés, jetons-nous dans la suite. Un jour, Savage-Rumbaugh, le frêle savant génial, alias la femme sauvage, redoutée de toute la profession, me dit: «Ici, c'est le laboratoire de Frankenstein, nous fabriquons des organismes ». Ce genre d'humour ne passe pas très bien devant les conseils d'administration ou chez les « pairs », mais Sue est incorrigible. Comme souvent, et dans l'interlocution orale infiniment plus encore que dans ses articles et livres, Savage-Rumbaugh commente avec brio et précision ce qui se passe et ce qu'elle fait. Après les honneurs

30. C'est bien souvent, je le crains, la position de Penny Patterson. 


\section{La grande scène des primates}

de l'institution (jusqu' au doctorat honoris causa à Chicago en 1997), elle est progressivement sortie du domaine de la science officielle, quittant plus tard l'université pour ce trust privé centré sur son expérience. Les textes qu'elle signe, et dont nous publions un exemple dans le dossier, relèvent désormais d'un champ épistémique singulier, agrégeant les connaissances dans un mépris royal des conventions de la science orthodoxe. Comme chez Frankenstein, c'est à un savoir hors normes, futur, interdit, prolifique, inacceptable que l'on a affaire. Ce n'est pas pour rien (ou pas que pour la plaisanterie) que Savage-Rumbaugh va chercher une comparaison dans le récit de Mary Shelley. On se livre ici à une expérimentation qui, agissant depuis le domaine de la science humaine et au-delà, communique avec une durabilité des questions théoriques et imaginaires, et passe aussi par l'écriture littéraire, une frénésie romantique, une épaisseur historique. À tel point que « les pairs » ne savent plus comment réagir à l'événement: après un certain cap, Savage-Rumbaugh entre dans ce qui reste pour l'instant invérifiable selon les standards d'une science professionnelle et organisée. Heureusement se trouvent d'autres manières de penser. Il n'est alors pas si indifférent qu'au moment de décrire sa créature, Victor Frankenstein use déjà d'une rhétorique comparable à celle de Tyson avec son Orang-Outan, sive Homo sylvestris ${ }^{31}$. Comme une circulation entre des régimes de vérité, de discours différents.

« J'avais du mépris pour les usages de la philosophie naturelle moderne. Tout était très différent lorsque les maîtres de la science cherchaient l'immortalité et le pouvoir; de telles vues, quoique futiles, étaient grandioses : mais maintenant la scène avait changé. L'ambition du chercheur semblait se limiter à l'annihilation de ces visions sur lesquelles était d'abord construit mon intérêt pour la science ${ }^{32} »$.

31. Le livre de Tyson paraît à Londres chez Bennet, en 1699. Laura Brown a souligné le rapport entre le portrait du démon dans le roman de Shelley et l'évocation des grands singes au dix-huitième siècle; cf. son Homeless Dogs \& Melancholy Apes, Ithaca, Cornell University Press, 2010, p. 59-63. 32. Mary Shelley, Frankenstein, or the Modern Prometheus, Londres, Whittaker, 1823, vol. I, p. 71. 
II est fort peu à parier que le langage, émergeant au sein d'individus animaux, sous les contraintes de leur environnement, de leurs formes de cognitions, de leur organisation sociale, de leurs possibilités organiques, soit en quelque manière un instrument parfait. Les écarts entre ce que je pense et dis, ce que je pense dire, ce que je dis penser, ce que tu comprends que je dis, ce que tu dis penser que je pense, etc., ces déplacements multipliés sont souvent partiellement neutralisés par les processus de corrections interactives, les indices contextuels, les habitus, les usages, les règles, etc. Dans plein d'occasions (celle du droit est exemplaire), la moindre déviation se trouve soudain si conséquente. Or la littérature est un art verbal créant de la signification en mobilisant ces manques et ces ambiguïtés du langage humain ordinaire.

Diogène le Cynique, dit-on, brandit un poulet plumé devant Platon pour ridiculiser la définition que ce dernier donnait de l'être humain comme « animal bipède et sans plume ${ }^{33}$ ». Au-delà des caractères physiques et génétiques d'Homo sapiens - un ensemble qui repose toujours sur la catégorisation de normalité tout en devant « contenir » les exceptions -, d'autres définitions de l'humain continuent d'avoir cours et nécessité pour les scientifiques, en particulier en psychologie expérimentale. Corpus impressionnant, foisonnant, où les plus grandes rigueurs méthodiques, stylistiques, conceptuelles et procédurales butent sur les effets de sens des mots employés - comme la sédimentation catégorielle. L'ignorance de la philosophie, hors le digest de l'étudiant et le blabla analytique standard, est en principe écrasante. Pour le mieux, bien souvent; une originalité de conception est à même de surgir là où une robotisante formation l'empêcherait. Et souvent pour le pire: reviendra pour la milliardième fois la bêtise de la tradition, sous les habits nouveaux du scientisme. Dans ces conditions, une définition verbale de l'être humain risque, malgré les précautions, de provoquer deux ou trois ambiguïtés, quelques regrettables incidents. Quant à éviter la grande célébration ou la redite déformante, encore moins joué.

Volontairement ou pas, les époux Gardner qui apprirent le langage des signes à la chimpanzé Washoe [45], et avant eux les Hayes essayant de faire parler la petite Viki [43], ou Witmer et Furness avec leur petite colonie simiesque [28] [32], ont tous reproduit le geste de Diogène. Ces biologistes ne faisaient pas qu'éprouver le rapport de proximité entre deux

33. Diogène Laërce, Vita, VI, 40. 
espèces de primates, pas que déterminer les potentialités à développer. Leur travail s'inscrivait en répons à une autre définition grecque de l'humain comme zôon logon ekhon, et regardait si par extraordinaire un autre animal, avec le langage, etc. Dans une perspective naturaliste, au sein de ce numéro, Savage-Rumbaugh et Fields associent à l'acquisition du langage un changement dans la conscience et la rationalité des bonobos, qui les met du côté des humains. Ils sont, je crois, fidèles à l'approche aristotélicienne, qui relevait de l'intersection entre la zoologie et la philosophie spéculative. Cette fidélité ayant les moyens d'une expérimentation que nul à Athènes n' aurait conçue ${ }^{34}$. Leur réponse est oui - sous l'index d'humain entrent tous les animaux dotés du langage humain, et, dès lors, d'une réflexivité, d'une logique, d'une inquiétude sans comparaison. Je ne dirais pas cela, ou ne le dirais pas comme cela, mais s'il faut se lancer dans la philosophie expérimentale ${ }^{35}$, ainsi que nous y invite aujourd'hui un courant renégat partant des départements de philo analytique, on ne trouverait pour un bon moment pas grand-chose de plus fondamental que cette enquête. Reste ensuite à comprendre, à interpréter.

Je veux reconnaître dans l'aventure des singes langagés plus qu'une tentative-limite en psychologie, plus qu'une pratique retardataire et dorénavant contraire à l'opinion des savants sur la nécessité de mesurer la cognition animale «telle qu'elle est», plus qu'une étape dans un cheminement d'histoire des sciences ${ }^{36}$. Je puis y trouver, via la condensation des temps et un agencement du théorique, un essai de fabrication prométhéenne, une expérience de modélisation du langage hors-support humain, un test métaphysique, une opération de fiction véridique aux bords d'un théâtre de la cruauté continuant toujours dans les risques les plus violents des corps, des paroles. Comme telle, la pointe avancée de ce mouvement, à savoir l'installation du Great Ape Trust, est apte à accomplir le conjectural d'hier sans ascendance directe. De même, c'est par la suggestion de Richard Yerkes [47], qui supposait possible en 1925

\footnotetext{
34. Je ne parle pas du fait que les grands singes y étaient sans doute inconnus.

35. Cf. Joshua Knobe et Shaun Nichols dir., Experimental Philosophy, Oxford, Oxford University Press, 2008.

36. C'est l'approche historique classique, illustrée par l'ouvrage aussi solide que limité de Gregory Radick, The Simian Tongue, Chicago, The University of Chicago Press, 2007. Ce même schème, mais dans le sens progressif et accumulatif, sous-tend l'interprétation que peuvent avoir certains expérimentateurs à l'égard de leur propre pratique ; cf. Sue Savage-Rumbaugh \& Robert Lewin, Kanzi, New York, Wiley, 1994, ou Roger Fouts \& Stephen Tukel Mills, Next of Kin, New York, Morrow, 1997.
} 
une acquisition partielle du langage par de grands singes grâce aux signes des sourds-muets, que les Gardner débutèrent. Mais en 1661, confronté à un grand singe, Samuel Pepys avait eu l'intuition d'enseigner à ce «monstre » si humain l'usage de «signes » [31]. Mais dès l'inauguration d'une instruction pour les sourds-muets, au cours du dix-huitième siècle, l'hypothèse avait surgi d'une application aux chimpanzés, aux orangs-outangs, dans telle provocation philosophique (La Mettrie), tel traité fumeux (Mondobbo), tel amusement semi-littéraire (Lignac) ${ }^{37}$. Avant l'oubli. Ces singes qui parlent, incroyablement présents, sont du passé reconstitué, notre avenir. Ils signalent la rigueur de la science, et la discipline d'un processus d'éducation, de processus d'établissements des résultats, comme un exil.

Je ne veux pas avancer, par l'appel à l'historique, au philosophique, qu'au fond tout se ramène (y compris la substance des sciences) à des schèmes factuels et chroniques, à des structures conceptuelles transcendantales. Je souligne que la part la plus forte des sciences est en contact avec ce qui lui échappe, et, par là, rencontre des discours dédiés à l'excès de connaissance. On ne résout pas une question philosophique par une expérience; au mieux, on la déporte. - En mobilisant la poésie, la fiction, je ne tente pas non plus de relativiser des résultats: je crois qu'il y en a, des « données », et je n'ai envie de les esthétiser afin de sauver quelque chose d'un échec programmé; nullement. Je ne prétends même pas que toute la science soit du discours, de l'institution sociale et qu'à ce titre, j'aie voix au chapitre, voire le dernier mot. Les sciences sont aussi cela, mais pas seulement. Autant l'événement que je décris, je le produis également, et autant son inconstructible m'attire, que je ne nierai ni ne négligerai aux seuls profits d'un beau mentir vrai.

37. Respectivement: [20], [27], [31]. 


\section{La grande scène des primates}

En neuro-imagerie, la variabilité, les erreurs, les signaux adventices sont étalonnés, négligés, comme, en pratique savante ordinaire, on nie l'incidence de l'opaque en se ramenant à une transparence fantasmatiquement totale. Une faible déviation dans la "signature " neurale associée à un mot entre plusieurs individus (voire «le même » en des instants différents) a des conséquences.

Je voudrais m'intéresser à ce que les scientifiques nomment le bruit, à ce qui échappe: I'en-plus pensé mentalement par rapport à la signature minimale et " commune », est-ce du sens, ou pas? L'hypothèse matérialiste ne devrait pas être en mesure de si légèrement écarter des productions neurales sous couvert qu'elles échappent à la statistique.

Non seulement « les sciences » mais nous avons du mal à raisonner sur le non-général. La conceptualité est déjà une tentation de tenir ensemble l'intenable ${ }^{38}$. La nomination porte avec elle un semblable mouvement d'arrêt, accréditant, plus qu'une aire sémantique, le souhait d'une fixité du sens et la subsomption du divers sous un mot donné. Les langages formels, la graphique, l'arithmétique, qui en sont venus à devenir les attributs visibles des « sciences », participent de ce projet. Le protocole expérimental, avec ses procédures, ses contrôles, indique encore la répétition sous le régulier. Cette pensée du général, potentiellement inscrite dans la cognition non verbale, est dans les mots néanmoins sans cesse martelée. À côté de ses réalisations, elle fait défaut dans l'ouverture de la signification, susceptible de fracasser la manœuvre de contention du noétique. La posture hétérologique des sciences est une manière supplémentaire de maintenir le général, plus strictement, plus proprement, que dans la seule diction langagière, qui n'est jamais complètement oblitérée (avec les conséquences induites ${ }^{39}$ ). Vraisemblablement, la généralisation est a priori favorisée dans les systèmes cognitifs animaux les plus développés. Sans avoir d'accès direct à l'inconstructible réel, notre appareil hypothético-déductif normal, notre rationalité qui subsume fonctionnent comme une excellente approximation; en général. L'épreuve d'autre chose dans la pensée, qui ne soit pas antérieur (l'indistinct de l'antéprédicatif) ou sans rapport, qui traverse la généralité en allant ailleurs, cette épreuve n'est évitée par aucune équation. Dans les autres voies du

38. Sur ce sujet, cf. mon article «Autant de non-réponses », à paraître dans la revue L'Autre.

39. Argument célèbre de Willard van Orman Quine, Word and Object, Cambridge (É.-U.), Technology Press of the MIT, 1960, § 33, p. 159 ; cf. $\$ 56$. 
savoir, en particulier les disciplines littéraires, les sciences humaines, qui sont faiblement ou aucunement hétérologiques, le choc et l'effraction du général sont davantage sensibles. Pour cette raison, et beaucoup d'autres, ces discours épistémiques ont privilégié des formes intermédiaires du général - le particulier, le spécial - tout en gardant plus de tendresse à l'égard de l'exception que la plupart des sciences. Là-dedans, que raconter, que mesurer, que décrire des singularités? comment affaiblir la généralisation sans verser dans l'idiotie? L'anecdotique de la science - ce fait qui pourrait dire beaucoup d'un coup, mais l'articule trop mal, à l'encontre des conventions, demeurant inexploitable - s'allie pour moi à une recherche de singularité, qui me porte au littéraire, au hors-norme, à l'extraordinaire. Je voudrais sérieusement penser avec les sciences (ce qui signifie retourner à l'école, recommencer, absorber les disciplines), et de préférence ce qu'elles ne pensent pas (car juste au-delà d'elles, en somme produit par elles), avec les moyens d'une connaissance poétique (qui œuvre, comme on sait en littérature).

Il est bien trop commode de se débarrasser des singes éloquents au nom de l'anecdote, de l'impossibilité à reproduire l'expérience, de l'imperfection des énoncés simiesques, de la part de folle fiction. Ces effets de la nescience à l'œuvre dans les sciences nous rappellent les tâches insurmontables, impossibles - celles qu'il vaut encore d'accomplir, en sus des simples et des simplement difficiles. 